\title{
Potential Solutions for Accessing Abstract Concepts in Embodied Theories of Cognition
}

\author{
Yijie Chen ${ }^{1 *}$, Yanyi $\mathrm{Wu}^{2}$, Kaiyi Wang ${ }^{3}$ \\ ${ }^{1}$ College of Education, University of Washington, Seattle WA 98195, United States \\ ${ }^{2}$ Zhengzhou No.7 High School, Zhengzhou, 450040, China \\ ${ }^{3}$ Weixun academy, Beijing, 100022, China \\ *Corresponding author. Email: cheny297@uw.edu
}

\begin{abstract}
The theory proved to be true is the embodied view, which illustrates that if people think about concrete concepts, such as 'kick' and 'pick,' they will use modality-specific simulations. However, abstract concepts posed a severe challenge for this embodied view. Then, researchers used mental metaphor to argue that people not only talk metaphorically but also think metaphorically, but this theory failed to explain if mental metaphors are embodied in modality-specific simulations. Therefore, in this paper, we search for other potential solutions and evaluate each of their supporting evidence.
\end{abstract}

Keywords: embodied cognition, abstract concepts, metaphor theory, moral cognition, social cognition

\section{INTRODUCTION}

For such a long time, researchers have tried to figure out how people think using their brains. Usually, the amodal theory is proved to play an important role in thinking, which illustrates that meaning is stored in brain areas that process amodal information. However, this theory is contradicted by convincing evidence of embodied view. In Pulvermueller's paper, he used three words 'kick, lick, and pick' to look for which region is activated when people read these three words [1]. Hence, the embodied cognition is proved to be correct during thinking: if people think about concrete concepts, they will use modality-specific simulations. This arouses another challenge to the embodied view: 'if thinking involves perceptual motor simulations, how can we ever think about abstract concepts that people cannot perceive from senses and act upon with the true movement, such as time, happiness, and so on [2].

For this challenge, two questions need be answered.

The first one is that if people think metaphorically, whether people use the source domain (perception) to think about the target domain (feelings). For this question, a mental metaphor can be the solution. For example, people often use space to think about time, putting their hands leftward to represent 'past' and rightward to represent 'future.' From the paper [3], people not only talk metaphorically but also think metaphorically because of people's cultural background. The experiment shows the strong association between leftward and pastward and rightward and futureward in thinking. The second question is whether the source domain is embodied in modality-specific areas. Researchers are seeking the answer and evidence for this question.

Firstly, in the paper [4], when using 'kick the ball,' there is overlap between activation in ROI and the activation during reading the phrase 'kick the ball', which is consistent with Pulvermueller's paper [1]. However, this overlap only presents for literal action, and no same

pattern occurs in metaphorical action when people read the phrase 'kick the bucket.' That is, there is no overlap between activation in ROI and the activation when the kick is used metaphorically. Therefore, this is the first failure that did not find the answer to the second question. After that, researchers tried to use another metaphorical spatial language: $\operatorname{good}=u p$, bad=down. In another paper [5], they use three different conditions. The first is that people use real space perception, and the other two are metaphorical spatial representations (positive and negative words). They use a computer model to examine what information in the brain distinguishes real higher and lower space. They want to 
find out which area in the brain this modal can succeed in identifying the metaphorical positive and negative space. The results showed that this modal only succeeded in SMGr and IPSI, representing the modalitynonspecific areas. After these experiments, we found that people indeed use space representing the source domain to think about good or bad and time representing the target domain, which answers the first question. However, no compelling evidence showed that the source domains are embodied.

The saying, "Absence of evidence is not evidence of absence", demonstrates that in situations where researchers cannot find evidence does not necessarily mean there is no evidence. Indeed, people failed to find out whether the mental metaphor is embodied using this way, but they were able to find other potential solutions to this question.

\section{BODY-SPECIFICITY HYPOTHESIS}

This hypothesis illustrates that 'If the content of our minds depends, in part, on how we interact with the environment... people with different kinds of bodies, who interact with the environment in different ways... should form correspondingly different concepts and word meanings' [2]. Hence, researchers assume that this hypothesis also adapts to abstract concepts. However, even if researchers believe that this can be a potential solution, it is true that they still do not find any compelling solution.

\section{EMBODIED MORAL COGNITION}

Embodied moral cognition is inspired by moral sentimentalism, which believes that human emotions and desires are essential to our understanding of morality, moral knowledge, and moral judgments. One of many versions of moral sentimentalism states that people's gut reactions create emotions, moral attitudes, and moral judgments first, while moral reasoning happens afterward as rationalization of these gut reactions [6,7,8]. In other words, embodied moral cognition holds that plenty of our moral judgments come from our embodied, affective states instead of abstract reasoning in our minds. Abstract concepts like moral decisions may be transformed into concrete concepts, expressed through physiological responses such as accelerated heart rate and sweating.

There are many sources of empirical evidence that can back up this point of view. Findings show direct evidence supporting the hypothesis that the vmPFC plays a significant role in promoting moral behavior through mediating the emotional processing of personal moral decisions [9]. In several pathological cases, studied patients such as psychopaths or individuals who have damages on the ventromedial prefrontal cortex (vmPFC) are impaired in making moral judgments - though psychopaths can hardly feel compunction about their immoral behaviors and may have difficulties differentiating moral from conventional norms from time to time [10]. Individuals with damage to the VmPFC area can preserve the knowledge of abstract moral principles but have a hard time making specific moral decisions in everyday life situations [11]. In both cases, tested individuals have a dearth of physiological responses along with neurotypical moral decision-making. With limited bodily experiences that guide moral judgments, these individuals incline to behave in more impulsive, selfish, and immoral ways. This suggests that humans may process abstract concepts in more means other than the metaphor theory. In the case of abstract concepts like moral decisions, our emotions and bodily reactions may be the key for us to access and understand them.

Embodied states can influence neurotypical individuals' moral judgments, as psychologists and neuroscientists have observed. For instance, neurovisceral integration may play a crucial role when people make moral judgments as visceral reactions and prefrontal regions seem to be integrated into moral judgments through outcome-based judgments [12]. If we experimentally manipulate one's perception of heart rate, we seem capable of influencing one's moral judgments.

Specifically, perception of faster heart rates results in feelings of a higher level of moral distress, leading to more just moral judgments [13]. Akin, there is also evidence showing that the existence of a feeling of disgust can lead to more rigorous moral judgments [14]. Disgust as a moralizing emotion demonstrates that it is regarded as evidence by participants that the act is wrong.

Researchers tend to endorse this claim despite their findings only giving correlational relationships rather than causal ones. What's more, contrary to feelings of disgust, which render people to make more severe moral judgments, when cleanliness is perceived, people tend to make less rigorous moral judgments [15]. These seem to support that perception of embodied cues mediates moral judgments. In addition, moral sentimentalists have observed that many people have solid aversive reactions to harmless actions that violate taboos, such as consensual protected sex between adult siblings, cleaning a toilet with the national flag, eating one's pet that had been run over, etc. In these cases, the strong negative affective response precedes the moral judgment, and often people have a difficult time articulating why they think these victimless, harmless actions are morally wrong $[6,16,17]$. From the perspective of embodied cognition, this ordering confirms the notion that we make moral judgments based on embodied cues.

While moral sentimentalism believes that all moral judgments are generated similarly, dual-process theories 
of moral psychology think otherwise. The existence of dual-process theories is to explain the seemingly inconsistent moral intuitions ordinary people have about moral dilemmas. Dual-process theories maintain that we have two systems in running moral decision-making. To be more specific, there is one system for Deontological reasoning and one for Utilitarian reasoning. As the former is driven by affect-less and abstract deliberation, the latter is driven by automatic, intuitive, emotional heuristics like gut feelings [18]. In the famous Trolley Problem, an out- of-control trolley is running on the trail with five people tied up on it; the only thing that the driver can do is to switch the railroad so that the trolley would go onto another track, but this track also has one person tied up on it. In this situation, most people would choose to switch the track, killing one person to save five people.

Nevertheless, if the situation changes a little bit, demanding that saving the five people need to push one person off and derail the trolley, most people would not choose to do so through this would result in the same consequences in the standard situation. The dual-process theory believes that in the former case, a System-2 type of abstract reasoning is responsible for our thinking, while in the latter case, our reasoning is guided by an aversive physiological response, or System-1 type, triggered by imagining the situation where we push a person off a footbridge [19]. The dual-process theory partially vindicates the moral sentimentalist view as it posits the System-1 type of moral reasoning based on embodied gut instincts. However, the difference is that it also believes in the existence of a separate system operating on different inputs and processes for more abstract moral reasoning.

Dual-process theories put reason and emotion on two different sides as if they oppose each other. Theorists have challenged this dichotomy recently [20,21,22] as they observe that there is an overlap in complex learning and inferential processing between the brain areas associated with emotions and the brain areas that impact individuals' ability to moral decision-making--individuals such as psychopaths or have damages in vmPFC areas who are impaired in this ability seem also suffer deficits in complex learning and inferential processing. This implies that abstract reasoning is not entirely cut off from affective processes. Quite the opposite, our expression of emotions through affective cues and physiological responses are indispensable to reasoning, learning, and decision-making. For the advocate of embodied moral cognition, this serves as further confirmation of the idea that all cognition, including moral cognition, is profoundly shaped by embodied cues [19].

\section{SOCIAL COGNITION-MIRROR NEURONS}

Social cognition enables humans to understand as well as interact with one another. This cognition involves attention, memory, affective cognition, and metacognition [23]. Although cognition involves numerous and diverse neuron processes, philosophers traditionally narrowly conceived social cognition as mentalizing to predict and explain human behaviors. However, embodied cognition theorists have rejected this narrow understanding of social cognition. Though they do not reject that neuron-typical adult can attribute beliefs and explain as well as predict behavior, they argue that this is a specialized and rarely used skill in our ordinary social interactions $[24,25,26]$.

Most social interactions require only essential underlying social cognitive capacities known as primary and secondary intersubjectivity. Primary intersubjectivity is the pre-theoretical, non-conceptual, embodied understanding of others that support the higher-level cognitive skills involved in mentalizing. It is "the innate or early developing capacity to interact with others manifested at the extent of perceptual experience-we see or more generally perceive in the other person's bodily movements, facial gestures, eye direction, and so on, what they intend and what they feel" [27]. The primary intersubjective exists once we were born and plays a crucial role in later adulthood, which manifests as the capacity for facial imitation, discerning and tracing eye movement, detecting intentional behavior, and "read" emotions from actions and expressive movements of others. At around the age of one, neurotypical children develop the capacity for secondary intersubjectivity. This development enables a subject to move from one-on-one, immediate intersubjectivity to shared attention. Based on

Embodied cognition, children learn practical skills when getting others to attend to an object and when learning to attend to objects others are attending to.

Thus, primary and secondary intersubjectivity are fundamental insofar as they are sufficient for navigating most typical social interactions and insofar as they enable the development of higher-level social cognition, like mentalizing. Moreover, this provides a better insight into abstract concepts involved in socializing that can turn into conceptual concepts and allow them to be learned as well as imitated without previous experiences of the concept. Mirror neurons activate both endogenously in producing behavior and exogenously in observing that very same behavior. Specifically, neurons in the premotor cortex and inferior parietal lobule activate when a subject uses them. These very same neurons selectively activate, and similar activation patterns are found when a subject observes a target interacting with an object. So, mirror neurons provide another thesis that we can understand another subject without physical or mental interactions. This helped to address the issue between abstract concepts and the 
function of the perceptual-motor neurons by suggesting it is possible to experience abstract concepts still. Though the interpretation of these findings is subject to a great deal of controversy, many theorists propose that mirror neurons are a fundamental mechanism of social cognition $[28,29,30]$.

In addition, the rationale is that mirror neurons explain how a subject understands a target's mental states without needing complicated, high-level inferences about behavior and mental states. In observation mode, the subject's brain activates as if the subject and target share the same feelings. Thus, observing the target's behavior is automatically meaningful to the subject, and mirror neurons are a possible mechanism for embodied social cognition. This finding suggests we can understand and interact with others without engaging in mentalizing. So, we can understand and experience abstract concepts through embodied cognition.

However, the claim above is more likely to be a prediction rather than a proven fact. So, more studies and more robust studies are needed to support this thesis to increase its reliability. Although some evidence supporting the theory and theory is reasonable and rational, the theory's lack of research and data is a significant issue. Lack of results generated from technical equipment vastly decreases the level of scientific as well as reliability. Therefore, with more and more vital data and relevant study, this theory could be one of the strong explanations to the abstract social concepts within the Embodied cognition and helps to overcome the conflict between perceptual neurons and abstract concepts.

\section{DISCUSSION}

For moral cognition, there are two general sides researchers discuss: moral sentimentalism and dualprocess theory. Moral sentimentalism comes from a physiological process. Experiments about abstract concepts have always focused on positive or negative moods and some specific moods, such as anger, fear, and disgust. So why do they choose disgust as a testbed?

Feelings of disgust can be shifted to objects with which they have nothing to do. Since disgust is related to nausea, it can easily trigger the nervous system. For a discussion of disgust, researchers focused on the effects of disgust on moral judgment for people susceptible to their own bodily experiences [14]. Their findings emphasized that emotional reactions are basically embodied [31]. Since disgust as a visceral feeling can induce nausea and some physical process for food expulsion, this kind of emotion consists of physical and embodied parts. However, it is claimed that disgust shows a more embodied aspect.
Hence, further study needs to investigate which kind of emotion is more embodied than the other one. Take sadness as an example. High and low on PBC in the sad situation showed the same meaning based on moral judgment. In addition, further study needed to focus on the individual's differences, such as their body consciousness, to investigate the relationship with the sensitivity of people's bodies.

For mirror neurons, they can be merely activated by certain kinds of action. Mirror neurons fire only when the monkey is shown with a natural action that aims at the object at the same time [32], for instance, when the researcher presents the "grasp" action for a piece of food in front of the monkey. They will not activate when people just move their hands but do not grasp something. Thus, mirror neuron activity can only be evoked by object-directed actions [33]. The claim of mirror neurons is more likely to be a prediction rather than proven facts. So, more studies and more robust studies are needed to support this thesis in order to increase its reliability.

Although there is some evidence to support the theory and the theory is reasonable and rational, the theory's lack of research and data is a significant issue. Lack of results generated from technical equipment vastly decreases the level of scientific as well as reliability. Therefore, with more and more factual data and relevant study, this theory could be one of the vital explanations to the abstract social concepts within the Embodied cognition and helps to overcome the conflict between perceptual neurons and abstract concepts.

\section{CONCLUSION}

This article illustrates the challenge, abstract concept, to the embodied cognition. For the first question researchers need to answer, the mental metaphor could be a solution to explain whether people think metaphorically. However, this theory had two failures for the second question. So, we explore further possible theories, the moral cognition and mirror neuron as social cognition, that may help explain how people think about abstract concepts in terms of embodied cognition.

\section{REFERENCES}

[1]. Pulvermuller, F. (2005) Brain mechanisms linking language and action. Nature Reviews Neuroscience, 6(7): 576-582.

[2]. Casasanto, D. (2009) Embodiment of abstract concepts: Good and bad in right- and left-handers. Journal of Experimental Psychology: General, 138: 351-367.

[3]. Casasanto, D. \& Jasmin, K. (2012) The Hands of Time: Temporal Gestures in English Speakers. Cognitive Linguistics, 23(4): 643-674. 
[4]. Aziz-Zadeh, L., Wilson, S.M., Rizzolatti, G., Iacoboni, M. (2006) Congruent Embodied Representations for Visually Presented Actions and Linguistic Phrases Describing Actions. Current Biology, 16(18): 1818-1823.

[5]. Ye, H.Y., Liu, X.Y., Liu, Y., Guan, Y.T. (2020) Peak Experience and High Altitude--Evidence for the Embodiment of Metaphor Theory. The Frontiers of Society, Science and Technology, 2(10): 49-53.

[6]. Haidt, J. (2001) The Emotional Dog and Its Rational Tail: A Social Intuitionist Approach to Moral Judgment. Psychological Review, 108(4): 814834.

[7]. Nichols, S. (2004) Sentimental Rules: On the Natural Foundations of Moral Judgment. Oxford University Press, Oxford.

[8]. Prinz, J.J. (2004) Gut Reactions: A Perceptual Theory of Emotion. Oxford University Press, Oxford.

[9]. Moretto, G., Làdavas, E., Mattioli, F., di Pellegrino, G. (2010) A psychophysiological investigation of moral judgment after ventromedial prefrontal damage. Journal of Cognitive Neuroscience, 22(8):1888-1899.

[10]. Hare, R.D. (1999) Without Conscience: The Disturbing World of the Psychopaths among Us. Guilford Press, New York City.

[11]. Damasio, A.R. (1994) Descartes' Error and the Future of Human Life. Scientific American, 271(4): 1094- 1144.

[12]. Park, G., Kappes, A., Rho, Y., Van Bavel, J.J. (2016) At the heart of morality lies neuro-visceral integration: lower cardiac vagal tone predicts utilitarian moral judgment. Social Cognitive and Affective Neuroscience, 11(10): 1588-1596.

[13]. Gu, J., Zhong, C.B., Page-Gould, E. (2013) Listen to Your Heart: When False Somatic Feedback Shapes Moral Behavior. Journal of Experimental Psychology: General, 142(2): 307-312.

[14]. Schnall, S., Haidt, J., Clore, G.L., Jordan, A.H. (2008) Disgust as Embodied Moral Judgment. Personality and Social Psychology Bulletin, 34(8): 1096-1109.

[15]. Schnall, S., Benton, J., Harvey, S. (2008) With a Clean Conscience: Cleanliness Reduces the Severity ofMoral Judgments. Psychological Science, 19(12): 1219-1222.

[16]. Haidt, J., Koller, S.H., Dias, M.G. (1993) Affect, Culture, and Morality, or Is It Wrong to Eat Your
Dog? Journal of Personality and Social Psychology, 65(4): 613-628.

[17]. Cushman, F., Young, L., Hauser, M. (2006) The Role of Conscious Reasoning and Intuition in Moral Judgment: Testing Three Principles of Harm.Psychological Science, 17(12): 1082-1089.

[18]. Greene, J.D. (2014) Beyond Point-and-Shoot Morality. Ethics, 124(4): 695-726.

[19]. Shapiro, L., Spaulding, S. (2021) Embodied Cognition. In: Edward N., Z. (Eds.), The Stanford Encyclopedia of Philosophy (Fall 2021 Edition). Stanford University, Stanford.

[20]. Huebner, B. (2015) Do Emotions Play a Constitutive Role in Moral Cognition? Topoi, 34(2): 427-440.

[21]. Maibom, H. (2010) What Experimental Evidence Shows Us about the Role of Emotions in Moral Judgement. Philosophy Compass, 5(11): 999-1012.

[22]. Woodward, J. (2016) Emotion versus Cognition in Moral Decision-Making: A Dubious Dichotomy. In: S. Matthew L. (Eds.), Moral Brains: The Neuroscience of Morality. Oxford University Press, Oxford. 87-116.

[23]. Fiske, S.T., Taylor, S.E. (2013) Social Cognition: From Brains to Culture. SAGE, London.

[24]. Gallagher, S. (2020) Action and Interaction. Oxford University Press, Oxford.

[25]. Gallagher, S. (2008) Inference or Interaction: Social Cognition without Precursors. Philosophical Explorations, 11(3): 163-174.

[26]. Hutto, D.D., Ratcliffe, M.M. (2007) Folk Psychology Re-Assessed.London: Springer, Dordrecht.

[27]. Gallagher, S. (2005) How the Body Shapes the Mind. Oxford University Press, Oxford.

[28]. Gallese, V. (2009) Mirror neurons and the neural exploitation hypothesis: From embodied simulation to social cognition. In: Jaimie. A. P. (Eds.), Mirror neuron systems: The Role of Mirroring Processes in Social Cognition. Humana Press, Totowa. 163-190.

[29]. Goldman, A.I. (2009) Mirroring, Mindreading, and Simulation. In Jaimie A. P. (Eds.), Mirror Neuron Systems. New York: Humana, New York City. 311-330.

[30]. Goldman, A.I., de Vignemont, F. (2009) Is Social Cognition Embodied? Trends in Cognitive Sciences, 13(4): 154-159.

[31]. Niedenthal, P.M., Barsalou, L.W., Winkielman, P., Krauth-Gruber, S., Ric, F. (2005) Embodiment in 
attitudes, social perception, and emotion. Personality and Social Psychology Review, 9(3):184-211.

[32]. di Pellegrino, G., Fadiga, L., Fogassi, L., Gallese, V., Rizzolatti, G. (1992) Understanding motor events: a neurophysiological study. Experimental Brain Research,91(1):176-180.

[33]. Caramazza, A., Anzellotti, S., Strnad, L., Lingnau, A. (2014) Embodied Cognition and Mirror Neurons: A Critical Assessment. Annual Review of Neuroscience, 37:1-15. 